ist dies auf keinen Fall und „richtige“ Fehler, Fehler die $\mathrm{zu}$ einem Wort im Blitzwörterbuch passen, wie etwa "dem“ statt „den“ werden nicht erkannt. Dies würde ein Verständnis der Satzstruktur voraussetzen, was aber von der derzeitigen Hard- und Software nicht erwartet werden kann.

Neben dem deutschen Blitzwörterbuch sind auch noch englische und französische zum Preis von je 221,- DM erhältlich. Damit weist das Programm Möglichkeiten auf, die man als Vorstufe zum „elektronischen Dolmetscher" bezeichnen könnte. Das Programm übersetzt Wort für Wort etwa vam deutschen in den französischen Text. Diese Art der Übersetzung ist alles andere als perfekt, aber unter Umständen mag es Bereiche geben, wo eine solche „Radebrecher-Übersetzung " ausreicht.

\section{Handbuch}

Zum Lieferumfang von WITCHPEN gehört auch schon in der Grundausstattung ein Handbuch, welches die Funktionen des Programmes sehr gut erklärt. Wie oben bereits erwähnt, bedeutet die Lektüre des Handbuches für diejenigen, die bereits mit WORDSTAR gearbeitet haben, keine Schwierigkeit. Das Handbuin besteht aus einem sehr aufwendig verarbeiteten Aufstellringordner. Die Lochung ist, wie in dieser Klasse üblich, kompatibel zu IBM-Ringordnern, ein Format, das sonst in Europa nicht erhältlich ist.

\section{Fazit}

WITCHPEN ist ein rundum gelungenes Textverarbeitungsprogramm. Die Rechtschreibkontrolle ist (mit den oben gemachten Einschränkungen) gelungen und kann auch von einem Einsteiger innerhalb kurzer Zeit erlernt werden. Bei intensivem Gebrauch von Rechtschreibkontrolle und Textkonstruktionen ergibt sieh ein großer Rationalisierungseffekt bei der Texteingabe. Die kleinen Mängel, die während des Tests festgestellt wurden, sind zwar ärgerlich, vergleicht man aber den Preis der Grundversion ven 112,- DM mit dem Preis anderer Textverarbeitungsprogramme, der regelmäßig weitaus höher liegt, so wiegt den finameielle Vorteil viele dieser Mängel auf. Gerade deswegen ist dieses Programm für den engagierten Jura-Studenten geeignet, der sich noch während seiner studentischen Laufbahn preiswert und komfortabel in die Textverarbeitung einarbeiten will.

Für fortgeschrittene Anwender, wie etwa wissen schaftlich tätige Juristen oder Anwaltskanzleien, ist dieses Programm nur bedingt zu empfehlen:

$Z$ wischenzeitlich ist eine neue Version von WITCHPEN erschienen, "WITCHPEN mal 5“, zurn Preis von 495,- DM. Das Grundmodul umfaßt nun neben der Textverarbeitung und der Programmiersprache auch noch eine Datenbank und eine Benutzeroberfläche mit Tastaturprogrammierung. Bei Gelegenheit soll auch diese erweiterte Version nach einem ausführlichen Test vorgestellt werden.

\title{
Informatik und akademische Jurisprudenz
}

(1)

\section{Maximilian Herberger}

\section{Teil 1: Versuch einer Bestandsaufnahme}

1. Die gegenwärtige Bedeutung der Informatik für die juristische Arbeit

A. Vorbemerkungen

1. Zwischen Bilanz und Perspektive

2. Bezugspunkt, Informatik": Theoretische Informatik als Instrument der Wissensverarbeitung

3. Bezugspunkt Jurisprudenz": Umgang mit in Texten ausgedrückten Informationen

a. Das Gewinnen von Text-Daten

b. Das Organisieren von Text-Daten

c. Das Analysieren von Text-Daten

d. Das Suchbarmachen von Text-Daten

e. Das Lehren und Lernen von Text-Daten

f. Das Fixieren von Text-Daten

g. Der Transfer von Text-Daten

B. Instrumente - Hilfsmittel — Arbeitsgebiete

1. Maschinelle Texterfassung
2. Textverarbeitung
3. Systeme zur Entscheidungsunterstützung
4. Statistikprogramme
5. Online-Datenbanksysteme
6. Microcomputergestuitzte Datenbanken
7. Volltextsysteme
8. Lemprogramme
9. Desktop Publishing
10. Computergestützte Buchproduktion
11. Elektronische Kommunikation

(Teil 2:

II. Konsequenzen für die Rechtsinformatik-Ausbildung
A. Vorüberlegungen
B. Vorschlag für ein Curriculum) 


\section{Die gegenwärtige Bedeutung der Informatik für die juristische Arbeit}

\section{A. Vorbemerkungen}

\section{Zwischen Bilanz und Perspektive}

Die Rechtsinformatik blickt mittlerweile auf eine etwa 20-jährige Geschichte zurück. Daten dieser Art sind Anlaß, „Bilanz und Perspektiven“ zu diskutieren. Die Aspekte „Bilanz“ und „Perspektive“ stehen für zwei mögliche (und nötige) Betrachtungsweisen. Beide Betrachtungslinien sind wichtig: Die erste zielt mehr auf eine Bestandsaufnahme, die zweite eher auf mögliche Folgen sich bereits andeutender Entwicklungen. Die anschließenden Überlegungen beziehen beide Aspekte ein. Dabei steht die praktische Vergewisserung über den bereits jetzt erreichten „Stand der Technik $^{\star}$ im Vordergrund, da sich auf dieser Grundlage die Überlegungen zur Entwicklungsperspektive hinsichtlich ihrer Realitätsnähe leichter beurteilen lassen. Allerdings ist eine derartige Bestandsaufnahme nur sinnvoll, wenn sie auch die kognitiven Auswirkungen ins Auge faßt, die sich aus dem neuen Umfeld der juristischen Arbeit ergeben. Denn andernfalls bliebe sie eine bloße Aufzählung von Geräten und Programmen, der jeglicher Orientierungswert fehlen würde.

\section{Bezugspunkt „Informatik“: Theoretische Informatik als Instrument der Wissensverarbeitung}

Eine Bestandsaufnahme der hier versuchten Art setzt Klarheit über die Bezugspunkte voraus. Zum Konzept der Informatik lassen sich einige Bemerkungen deswegen nicht umgehen, weil außerhalb der Informatik dazu nicht immer ein klares Bild existiert. Eine verbreitete Fehlvorstellung identifiziert „Informatik“ mit „Computerwissenschaft ${ }^{\star}$ in dem Sinne, daß der Computer als Maschine dabei im Mittelpunkt steht. Diese Perspektive verkennt, daß die technische Informatik (die sich grob als maschinenorientiert beschreiben ließe) nur ein Teilgebiet der Informatik ist. Hinzu kommt u.a. die theoretische Informatik, die sich prinzipiell als maschinenunabhängig konzipieren läßt (obwohl der Computer sich auch hier als wertvolles Hilfsmittel erweist). Es ist zwar gegenwärtig zugegebenermaßen so, daß Informatik-Wissen seinen Weg in andere Disziplinen meist über den Einsatz bestimmter Hard- und Software-Produkte findet. Deswegen gruppiert die folgende Übersicht auch nach derartigen Instrumenten und Hilfsmitteln, die bereits jetzt von Juristen genutzt werden. Man sollte dabei aber nicht übersehen, daß der wahrscheinlich viel tiefer reichende Rezeptionsweg über die Integration von Methoden der theoretischen Informatik in die Rechtstheorie verlaufen dürfte (und sollte). Der Dialog dazu ist in der Bundesrepublik noch kaum in Gang gekommen. (2) Schon jetzt läßt sich aber absehen, daß die von der theotetischen Informatik (als Teil der „cognitive science“) angebotenen Methoden der Wissensverarbeitung (und richtig verstanden - auch der Informationsverarbei- tung) dem juristischen Denken nicht nur nicht fremd sind, sondern sich mit analytischen Instrumenten der juristischen Tradition berühren.

\section{Bezugspunkt ,Jurisprudenz“: Umgang mit in Texten ausgedrückten Informationen}

Selbst wenn man anknüpfend an Berührungspunkte in der Praxis eine Analyse der vorliegenden Art nach Informatik-Instrumenten und -Hilfsmitteln gliedert, setzt eine systematisch orientierte Bilanz mindestens umrißartig auch eine Vorstellung von der juristischen Berufsrolle voraus, innerhalb derer sich die im folgenden darzustellenden Arbeitsbereiche sinnvoll zusammenfügen lassen.

Die wissenschaftliche Arbeit des Juristen konzentriert sich auf in Text-Daten ausgedrückte Informationen. Der innerhalb der „cognitive science“ zentrale Gesichtspunkt der Informationsverarbeitung erweist sich so als geeigneter Aspekt zur Behandlung dieses Teils juristischer Tätigkeit. Bezogen auf den dadurch umrissenen Gegenstandsbereich sind die im folgender genannten Operationen integraler Bestandteil der Arbeit des Juristen. Diesen Techniken (im Sinne des alteri techne-Begriffs) kann man die neuen Techniken in zwangloser Weise zuordnen, was für die systematische Tragfähigkeit des Ansatzes spricht.

Die (auch) in der juristischen Arbeit anzutreffenden Grundformen des Umgangs mit Textdaten sind (jeweilssmit zugehöriger Informatik-Technik):

\section{a. Das Gewinnen von Text-Daten}

Was das Rohmaterial an Text-Daten angeht, kann man (sofern man diese für die EDV-Weiterverarbeitung erfassen will) auf maschinelle Texterfassungssysteme zurückgreifen.

(Vgl. dazu den Abschnitt „Maschinelle Texterfassung ${ }^{*}$ )

\section{b. Das Organisieren von Text-Daten}

Die Organisation von Textdaten (beschränkt auf den Aspekt der Einbettung in Lesetexte) wird mittlerweile in schon fast selbstverständlicher Weise als die Domäne der Textverarbeitung betrachtet.

(Vgl. dazu den Abschnitt „Textverarbeitung“.)

(1) Die Arbeit hat dem Wissenschaftsrat als Gutachten vorgelegen. Sie ist, was die Bilanz der Rechtsinformatik-Aktivitäten an den deutschen juristischen Fakultäten angeht, wegen der zwischenzeitlichen erfreulichen Entwicklung nicht mehr ganz vollständig. Da der Abdruck jedoch im wesentlichen Dokumentationszwecke verfolgt, wurde auf eine diesbezügliche Aktualisierung verzichtet. Es kann insoweit auf den demnächst erscheinenden, von Eberle herausgegebenen Protokollband zur Hamburger CIP-Tagung 1988 verwiesen werden.

(2) Ein gelungener erster Versuch war der vom Arbeitskreis „Formalisierung und Expertensysteme im Recht" der GI veranstaltete Workshop „Expertensysteme im Recht Auswirkungen auf Rechtstheorie und Computerrecht ${ }^{\star}$ (Tübingen, 25./26.Juni 1987). 


\section{c. Das Analysieren von Text-Daten}

Soweit es um die formal beschreibbaren Relationen von Textdaten geht, bilden die für die Konstruktion von „Expertensystemen“ verfügbaren Hilfsmittel geeignete Arbeitsinstrumente. Die durch deren Hilfe erleichterte Analyse ist auch die Voraussetzung dafür, einen Text in suchbarer Form zur Verfügung zu stellen (vgl. dazu im folgenden den Punkt „Die Erschließung von Textdaten für die Suchec).

(Vgl. dazu den Abschnitt „Systeme zur Entscheidungsunterstützung ${ }^{*}$.)

Wenn statistische Auswertungen von Text-Daten gefragt sind, kann man auf Statistikprogramme zurückgreifen. Unter Umständen bildet die statistische Auswertung auch die Voraussetzung für geignete Suchstrategien im Fext (vgl. dazu im folgenden den Punkt „Text-Daten suchbar machen“). Darüberhinaus sind Statistikprogranmme tuatürlich auch für den soziaìempirisch arbeitenden Juristen hilfreich.

(Vgl. dazu den Abscanitt „Statistikprogramme ${ }^{\star}$ )

\section{d. Das Suchbarmachen von Text-Daten}

Große Textmengen sind ohne Aufbereitung für die Suche (information retrieval) nictit handhabbar. Diese bereits bei gedrucktem Material geläufige Erfahrung gilt verstärkt für „elektronische Literatur“. Datenbankund Volltextsysteme bieten sich hier als Unterstützung für die Aufgabe der Informationssuche an.

(Vgl. dazu die Abschnitte „Online-Datenbanksysteme“, „Mikrocomputergestützte Datenbauken“, „Volltextsysteme ${ }^{\star}$.)

\section{e. Das Lebren und Lernen von Text-Daten}

Die Aufgabe, juristische Informationen lerngeeignet aufzubereiten, hängt eng mit dem bereits erwähnten Aspekt der Analyse von Text-Informationen zusanımen. Da beim Konzipieren von Lernmaterial aber noch zusätzliche didaktische Anfordeningen hinzukommen, handelt es sich um eine eigenständige Tätigkeit, die sich nioht it der Analyse erschöpft. Dementsprechend hat sich der Sektor der "Lernprogramme (auch „courseware“ oder CAL - für ${ }_{n}$ computer assisted learning ${ }^{\alpha}$ - genannt) mit guten Gründen als eigenständiges Gebiet etabliert.

(Vgl. dazu den Abschnitt „Lernprogramme“.)

\section{f. Das Fixieren von Text-Daten}

Die Publikation bildet normalerweise den Abschluß wissenschaftlicher Arbeit. Unter den Einfluß neuer Technologien haben sich auch die Publikationsformen gewandelt. Das sog. „Desktop publishing ${ }^{\star}$ stellt eine kostengünstige Art des Publizierens vom Arbeitsplatz aus dar.

(Vgl. dazu den Abschnitt „Desktop Publishing“.)

Weiterhin ist der Weg aus der Textverarbeitung in den Fotosatz als eine das Umfeld der Buchproduktion nachhaltig verändernde Arbeitsmethode hier anzusiedeln.

(Vgl. dazu den Abschnitt „Computergestützte Buchproduktion “.)

\section{g. Der Transfer von Text-Daten}

Die bisherige Distribution wissenschaftlicher Ergebnisse beschränkte sich - abgesehen von der mündlichen Kommunikation (wozu auch Rundfunk und Fernsehen rechnen) - auf die Druckmedien. Dazu treten jetzt die Möglichkeiten der elektronischen Übermittlung von Texten. Diese Texte sind dann beim Adressaten so verfügbar, daß er sie in einer der aufgezählten Weisen nutzen und bearbeiten kann.

(Vgl. dazu den Abschnitt „Elektronische Kommunnikation".)

Unter allen genannten Arbeitsaspekten bkdienen sich Juristen der von Seiten der Informatik angebotenen Hińsmittel. Allerdings gibt es keine breite empirische Bestandsaufnahme zur universitären Situation auf diesem Sektor. Eine „Delphi-Studie“, wie sie etwa zur Computerlinguistik durchgeführt wurde, wäre deshalb sehr wünschenswert. Immerhin existiert eine Übersicht zum „rechtsinformatischen“ Veranstaltungsangebot. (3) Weitere Aufschlüsse sind von der demnächst tagenden Konferenz der Organisatoren juristischer CIP-Pools zu erwarten. Trotzdem muß die hier gegebene Übersicht mit dem Vorbehalt versehen werden, daß sie nur auf informellen Umfragen ohne Anspruch auf Vollständigkeit beruht. (4)

\section{B. Instrumente - Hilfsmittel - Arbeitsgebiete}

\section{Maschinelle Texterfassung}

Mit „maschineller Texterfassung“ ist hier nicht die manuelle Texteingabe in ein Computersystem gemeint (vgl. dazu den Abschnitt „Textverarbeitung“). Vielmehr wird darunter die maschinelle Transformation gedruckter Texte in ein maschinenlesbares Format verstanden. Speziell für Juristen ist diese Technologie von Interesse, weil die datenbankmäßige (vgl. dazu den Abschnitt „Datenbanken“) Aufbereitung gedruckt vorliegenden Rechtsprechungsmaterials, an deren wissenschaftlicher Notwendigkeit kein Zweifel besteht, oft an den beschränkten Erfassungsmöglichkeiten scheitert. Gleiches gilt etwa für das Korpus der Allgemeinen Geschäftsbedingungen. Diese ungünstige Ausgangslage ändert sich, sobald man nicht mehr auf manuelle Erfassung angewiesen ist.

Technologisch wutde im Gebiet der Zeichenerkennung bisher der Standard durch die KDEM (Kurzweil Data Entry Machine) gesetzt. (5) Dieses Gerät ist trotz des damit verbundenen großen Investitionsvolumens im geisteswissenschaftlichen Bereich schon verschie-

(3) Wolfgang Heinz, Iudex non calculat oder: Weshalb soll und wie kann den Juristen die Angst vor dem Computer genommen werden?, Informatik und Recht, 1987, S. 242-247, 244, mit umfassender Bibliographie zum Thema.

(4) Eine gewisse Vollständigkeit ergibt sich vielleicht daraus, daß der Versuch einer Gesamtorientierung mit der redaktionellen Arbeit an der Zeitschrift "Informatik und Recht" verbunden ist.

(5) Vgl. dazu Maximilian Herberger, Die Maschine die liest, Rechtshistorisches Journal, Bd. 2(1983), S. 227-233. 
dentlich im Einsatz. Auch juristische (vor allem rechtshistorische) Projekte werden damit bearbeitet. (6) Allerdings setzten die relativ hohen Kosten der KDEM einer weiteren Verbreitung Grenzen. In den letzten Monaten hat sich hier ein grundlegender Wandel ergeben. $\mathrm{Zu}$ den jetzt sehr preiswerten Scannern ist leistungsfähige Zeichenerkennungssoftware verfügbar, die maschinelle Texterfassung am einzelnen Arbeitsplatz in greifbare Nähe rückt. (7) Zwar gibt es bisher im juristischen Bereich (abgesehen von vereinzelten Tests) noch keine breitere Resonanz auf diese Systeme. Das dürfte sich aber ändern, wenn diese neuen Instrumente besser bekannt sind. Deren breite Nutzung wird es dann erlauben, eine Materialbasis zu schaffen, die zusammen mit leistungsfähigen Datenbanksystemen ein Mehr an Rechtsstoff in gezielterer Weise als bisher zugänglich macht.

\section{Textverarbeitung}

Die mikrocomputergestützte Textverarbeitung ist mittlerweile an den meisten juristischen Fakuftäten auf die eine oder andere Art etabliert. Dabei gehört zur Textverarbeitung auch das automatische Erstellen von Inhaltsverzeichnissen, die Fußnotenverwaltung und das Generieren von Wort- bzw. Paragraphenlisten. Zwar gibt es, wie die Vorworte mancher Veröffentlichungen dokumentieren, noch immer das Marruskript, das in mehrfachen Versionen auf der Schreibmaschine geschrieben wird, und zu dem dann ausschließlich manuell Inhaltsverzeichnis und Register erstellt werden. Doch befindet sich diese Form der Manuskripthenstellung unaufhaltsam auf dem Rückzug. Angesichts dieser Situation stellt sich die Frage, ob man es dabei nur mit einem Wandel im arbeitstechnischen Bereich zu tun hat, oder ob die juristische Arbeitsweise in irgendeiner Form betroffen ist. Mehrere Gründe sprechen dafür, in diesem Wandel mehr als eine „bloß technische“ Veränderung zu sehen.

Erstens verändert sich das Verhältnis des Autors zum Text. (8) Der Text ist für ihn nicht mehr in derselben Weise wie bisher statisch fixiert. Vielmehr präsentiert er sich als flexibles Material, das den vielfältigsten Umorganisationen zugänglich ist. Das gilt allerdings im vollen Sinne nur, wenn der Autor selbst den Text am Computer bearbeitet. Das bedeutet nicht notwendig, daß er auch selbst die Ersterfassung vorgenommen hat. Diese kann z. B. nach wie vor auf Grund eines Diktats erfolgen. Wenn der Autor aber stets nur mit Ausdrukken arbeitet und sich nie selbst an den Rechner begibt, verschenkt er die Möglichkeit, dem Text in der skizzierten Weise neu zn begegnen.

Zweitens (und das hängt mit dem eben genannten Aspekt zusammen) stellen fortgeschrittene Textverarbeitungsprogramme den Text in einer Struktur zur Verfügung, der ihn nicht mehr als bloß lineare Abfolge von Zeichen erscheinen läßt. Dies beginnt schon dort, wo das Textverarbeitungsprogramm die Verwaltung der Textgliederung übernimmt. Unter dieser Voraussetzung ist der Text auch in gliederungsbezogenen Einheiten vorhanden, die entsprechend umarrangiert werden können. Eine entscheidende Weiterentwicklung in dieser Richtung stellen die (z.T. schon in Textverarbeitungsprogramme integrierten) sog. „OutlineProzessoren“ dar, die eine Vielzahl von gliederungsbezogenen Operationen erlauben. So kann man etwa bestimmte Text- und Gliederungsebenen ausblenden, um nur das „Skelett“ des Textes zu betrachten. Auf diese Weise gewinnt der Text eine neue Flexibilitä der Struktur, die kreative Möglichkeiten eröffnet.

Drittens schließlich entsteht durch den umfassenden Einsatz der Textverarbeitung eine Situation, in der alle wissenschaftlichen Manuskripte auch in "elektronischer $^{\star}$ Form vorliegen, bevor sie in irgendeiner Form (aus-)gedruckt werden. Die in letzter Zeit aufgekommenen Überlegungen, eine umfassende wissenschaftliche Textdatenbank entstehen zu lassen, bauen auf dieser Infrastruktur auf. (9)

Wie sieht nun - gemessen an den skizzierten Möglichkeiten - die Bilanz auf dem Gebiet der Textverarbeitung aus? Weil man vielfach die Textverarbeitung noch rein instrumentell betrachtet (etwa i.S.d. Schlagworts von der „besseren Schreibmaschine“), hat sich ein gewisser verhängnisvoller Kreislauf eingestellt, der die Textverarbeitung auf diesem niedrigen Nivean festschreibt. Das hängt - wie bereits angedeutet - auch damit zusammen, daß vielfach die Textverarbeitung insgesamt delegiert wird und der Wissenschaftler sie zu keinem Zeitpunkt inı Prozeß der Textherstellung selbst einsetzt. Es dürfte allerdings nur eine Frage der Zeit sein, bis sich auch der Arbeitsplatz des juristischen Wissenschaftlers so umgestaltet hat, daß der Mikrocornputer selbstverständlich dazu gehört. Dann werden die eben beschriebenen Folgen, die bereits jetzt in einigen Bereichen zu beobachten sind, generell eintreten. Unter dem Aspekt der praxisorientierten Bestandsaufnahme läßt sich aber noch nicht konstatieren, daß die innovativen Textverarbeitungskonzepte adäquat ausgeschöpft würden.

\section{Systeme zur Entscheidungsunterstützung}

Die bisher erwähnten Anwendungen waren textbzw. datenorientiert, ohne die logische Struktur der im Text enthaltenen Regeln ins Auge zu fassen. Bei Systemen zur Entscheidungsunterstützung hat man es mit

(6) $\mathrm{Zu}$ nennen sind hier vor allem die Arbeiten des Max Planck-Instituts für europäische Rechtsgeschichte (Frankfurt/Main), wie etwa auch das Projekt zur Erfassung der verbreitetsten AGB's (Manfred Wolf/Frankfurt).

(7) Hierher gehört das von der Akademie der Wissenschaften (Mainz) gegenwärtig für die Erfassung kyrillischer Vorlagen (Projektleitung: Dr. Schulz) eingesetzte System OPTOPUS.

(8) Vgl. dazu die kurzen aber treffenden Bemerkungen bei Gregor/Krifka, Computerfibel für die Geisteswissenschaften, München 1986, S. $62 \mathrm{f}$.

(9) $\mathrm{Zu}$ denken ist hier in erster Linie an die entsprechende Initiative des Nomos-Verlages. Eine solche Textdatenbank erlaubt es auch, das „nachfrageorientierte Publizieren“ (publishing on demand) auf eine geeignete organisatorische Grundlage zu stellen. 
regelprientierten Programmen zu tun. Die gegenwärtig wichtigste Teilklasse bilden die sog. „Expertensysteme (wissensbasierten Systeme), die sich dadurch auszeichnen, daß sie eine Deduktionskomponente beinhalten. Im deutschen juristischen Bereich existieren dazu erst Prototypen, die allerdings die Leistungsfähigkeit derartiger Systeme schon insoweit demonstrieren, daß man die Möglichkeit praktiscber Arbeitsinstrmmente dieser Art als realisierbar betrachten muß. (10) Es läßt sich nicht bestreiten, daß die juristische Tätigkeit int weiten Bereichen darin besteht, Regeln $\mathrm{zu}$ formulieren, die Struktur von Regeln zu analysieren und die Verknüpfungen von Regeln zu betrachten. Angesichts der normalen Komplexität auch kleinener juristischer Teilsysteme ist dieses Programm „mit Papier und Bleistift ${ }^{\text {" }}$

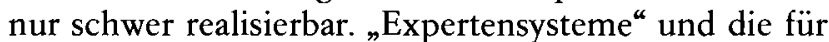
deren Programmierung zur Verfügung stehenden Hilfsmittel erlauben es, ein Mehr an Regelkomplexität im analytischen Zugriff zu haben. Sie erweisen sich damit als "Denkverstärker". Zugleich führt die Interaktion mit dem vom juristischen Experten selbst zusammengestellten Regelwissen dazu, daß dieses in einer auf ungewohnte Weise verselbständigten Form dem menschlichen Wissensträger gegenübertritt. Die sich daraus ergebene neue Dimension der Wissenshandhabung weist allerdings weit in dfe Zukunft und hat noch keinen breiten Rückhalt in der gegenwärtigen juristischen Wissenschaftspraxis.

Neben den „Expertensystemen“ existieren auch Programme zur Entscheidungsunterstützung, die keine Deduktionskomponente integrieren. Diese Programme beschränken sich darauf, eine (meistens baumartige) Strukturierungsmöglichkeit anzubieten, innerhalb derer das Wissen eines Bereichs arrangiert werden kann. Der Entscheidungsweg besteht dann darin, diesen Baum zu durchlaufen. Dabei können auch Alternativen verglichen werden. Versieht man die Knotenpunkte des Baumes mit Wahrscheinlichkeitskoeffizienten, läßt sich das Entscheiden unter Unsicherheit abbilden. (11) Was eben zu den Konsequenzen der Anwendung von „Expertensystemen“ gesagt wurde, gilt hier entsprechend.

\section{Statistikprogramme}

Soweit der Jurist mit Methoden der empirischen Sozialforschung arbeitet, wie dies etwa in der Rechtssoziologie und der Rechtstatsachenforschung der Fall ist, setzt er statistische Methoden ein. Auch hier bieten die mittlerweile seinon itn Mikrocomputerbereich vorhandenen Statistikprogramme entscheidende Unterstützungsmöglichkeiten, die von den auf diesem Gebiet tätigen Forschern auch ausgeschöpft werden. Angesichts des Bekanntheitsgrades statistischer Arbeitsweisen genügt dieser Hinweis zur Charakterisierung des dort gegebenen Standes der Technik. Hinzuzufügen ist allerdings, daß sich die statistischen Auswertungsmöglichkeiten in analytisch weitreichender Weise mit don "Textdaten “ in Verbindung bringen lassen, die aus den eingangs genanmten Gründerl in immer breiterem Maße im Computer vorhanden sind. Auch hier wird wieder der integrative Trend sichtbar, der darauf beruht, daß man es im Grunde in allen der verschiedenen beschriebenen Arbeitssparten mit Wissensverarbeitung zu tun hat. Es ist beispielsweise denkbar, über Worthäufigkeitsanalysen zu einer vorläufigen automatischen Indexierung von Texten zu kommen. Damit das erfolgreich sein kann, muß man allerdings die für diese Textdaten geeigneten statistischen Anakysen vornehmen. In ähnlicher Weise versucht man, die Datenbankrecherche dadurch zu unterstützen, daß statistisch identifizierte Wort-Cluster als Suchindikatoren verwendet werden. (12)

\section{Online-Datenbanksysteme}

In der Bundesrepubrik Deutschland sind mehrere juristische Datenbanken etabliert. $\mathrm{Zu}$ nennen sind (klammert man die Datenbanken mit jurisrischen Angeboten im Beiprogramm aus): (13)

- juris

- CELEX

- Lexinfornı

- Asyldoc

Lexinform richtet sich weitgehend an die steuerberatenden Berufe. Asyldoc deckt den Spezialbereich des Asylrechts $a b$ and ist deswegen vom Inhalt her für eine kleinere Benutzergruppe gedacht. (14) CELEX ist umfassend euraparechtlich orientiert, dadurch aber zugleich auch spezialisiert. Damit verbleibt als einzige juristische Datenbank zum deutschen Recht mit generellem Anspruch juris. Hier hat sich von der breiteren juristischen Öffentlichkeit noch weitgehend unbemerkt eine Nutzungsform herausgebildet, die dieser Datenbank eine rege Naeinfrage verschafft. Gemeint sind die an den verschiedenen Universitätsbibliotheken existierenden Informationsvermittlungsstellen, die zum Teil zu ermäßigten Gebühren juris-Recherchen ermögli-

(10) Genannt seien für die Bundesrepublik das Tübinger LEX-Projekt und MULE (Munich Legal Expert System). Vgl. zum Stand der juristischen Expertensysteme in der Bundesrepublik Deutschland, der Schweiz, den Niederlanden und Belgien Ulrich Erdmann/Herbert Fiedler/Fritjof Haft/Roland Traunmüller (Hrsg.), Computergestützte Juristische Expertensysteme, Tübingen 1986. S. ergänzend noch Fiedler, Orientierung über juristische Expertensysteme, CR 1987, S. 325-331.

(11) Eine aktuelle Übersicht über „decision support systems“ findet sich im September-Heft 1987 von PC-Magazine.

(12) Vgl. dazu D.L.Pape, Speedier Processing and Escaping from Boolean Logic, in: Colin Campbell (Hrsg.), Data Processing and die Law, London 1984, S. 73-89.

(13) Vgl. als knappen Gesamtüberblick (Stand allerdings 1984) Svoboda, Juristische Informationssysteme. Ein Überblick über deutsche und ausgewählte europäische Systeme sowie Forschungsaktivitäten und internationale Komitees, München 1984.

(14) Allerdings ist das Datenbank-Konzept von ASYLDOC methodisch von Interesse, vor allem was die Integration mikrocomputergestützter Datenbanken in eine OnlineUmgebung angeht. Vgl. zu ASYLDOC IuR 1986, S. 85-86, 117-122, 172-174. 
chen. (15) Manche dieser Informationsvermittlungsstellen sind durch die Nachfrage von studentischer Seite aus auf Wochen hinaus im voraus ausgebucht. (16) Für das juristische Examen wird juris von vielen Kandidaten schon mit Selbstverständlichkeit benutzt, wie sich auch daran ablesen läßt, daß verschiedene Justizprüfungsämter sich veranlaßt sahen, dazu Benutzungsvorschriften herauszugeben. Inwieweit die akademische Jurisprudenz juris tatsächlich ausschöpft, läßt sich mangels empirischer Untersuchungen dazu nicht präzise sagen. Es ist jedoch zu vermuten, daß gerade die Literaturdatenbank mit 660 ausgewerteten Zeitschriften ein Informationsangebot darstellt, das sich auf Dauer nicht ausklammern läßt.

\section{Microcomputergestützte Datenbanken}

Ebenso wie die Textverarbeitung auf dem Mikrocomputer gehört auch der Aufbau eigener Datenbanken auf dem Mikrocomputer zum verbreiteten „Stand der Technik“ im juristischen Bereich. Vermutlich wird sich in naher Zukunft sogar eine Integration von Textverarbeitung und Datenbanksystemen auf der Programmebene einstellen. Zahlreiche Juristen in Universität (und auch Justiz) haben mit Hilfe am Markt verfügbarer Datenbankprogramme eigene Datenbanken zu Rechtsprechung und Literatur aufgebaut. Abgesehen von dem Rationalisierungsaspekt, der auch hier zum Tragen kommt (man denke etwa an die Neuauflage von Büchern), beeinflußt dieser Trend mït gewichtigen kognitiven Folgen die Form, in der juristisches Wissen verfügbar ist. Es liegt nicht mehr nur sequentiell für den punktuellen Zugriff aufbereitet vor, sondern kann nach den gewählten Strukturierungskriterien beliebig gruppiert werden.

Es stellt sicher eine zutreffende Diagnose dar, wenn man behauptet, daß kleinere mikrocomputergestützte Datenbanken der eben beschriebenen Art im juristischen Bereich als Arbeitsinstrumente etabliert sind. Trotzdem ließ sich bis vor kurzem nicht übersehen, daß es sich hier um heterogene Projekte einzelner Juristen handelte. Ein Projekt mit Breitenwirkung war nicht ersichtlich. Das dürfte sich jetzt durch die von vier BGH-Richtern betreute Datenbank BGH-DAT ändern. Diese für Mikrocomputer publizierte Datenbank bietet die Leitsätze und zusätzliche strukturierte Informationen für alle BGH-Urteile. Die Lieferung für 1986 liegt vor. Beroits eine vorläufige Betrachtung zeigt, daß mit einem solchen, zur gedruckten Sammlung hinzutretenden Instrument die juristische Informationsverarbeitung auf ein neues Niveau gehoben werden kann. Ob dies der Fall sein wird, hängt allerdings auch davon $a b$, ob die juristische Argumentationstheorie diese neue Form der vertieften Materialerschließung (etwa was das Wortlaut-Argument angeht) integriert.

\section{Volltextsysteme}

Bei den Textverarbeitungsprogrammen war bereits andeutungsweise die Rede davon, daß diese durch
Suchmöglichkeiten den Text in einer neuen Form zugänglich machen. Allerdings handelt es sich dabei nur um eine lineare Suche, die sich angesichts komplexerer Suchwünsche sofort als unzureichend erweist. Auf diese Lücke zielen Volltextsysteme, die mit komplexen Indexierungsmechanismen den gesamten im Computer gespeicherten Text auch für Suchfragen mit logischen Verkmüpfungen zugänglich machen. Diese im amerikanischen Bereich bereits in breiterem Maße eingesetzten Volltextsysteme werden jetzr auch an verschiedenen deutschen Universitäten erprobt. (17) Ihr Vorteil besteht im Sinne des bereits mehrfach unterstrichenen Trends darin, den Text in einer von der sequentiellen Struktur abweichenden Weise zugänglich zu machen. Dabei unterscheiden sich diese Volltextsysteme von den oben beschricbenen mikrocomputergestützten. Datenbanksystemen dadurch, daß sie keine feldorientierte Strukturierung der Information verlangen. Vielmehr ist jedes Wort des Textes in logischer Verknüpfung (u.U. modifiziert durch Abstandskriterien) ein denkbares Suchziel. Im Sinne der Integration zwischen bisher getrennten Programmsparten werden diese Volltextsysteme auch auf Mikrocomputern mit feldorientierten Datenbanksystemen kombiniert. (18) Es ist bereits jetzt möglich, Texte mit Feldern variabler Länge und Textkomponenten variabler Länge so zu speichern, daß die feldorientierten Suchmöglichkeiten und die Freitextsuchmöglichkeiten gleichzeitig gege ben sind. Diese für juristischen Anwendungen optimale Arbeitsumgebung wird bereits bei einigen Projekten exprobt, kann aber noch nicht als etablierte Technik mit Breitenwirkung angesprochen werden. Die oben erwähnte Datenbank BGH-DAT ist ein Schritt in diese Richtung, da das dort verwendete Datenbanksystem sehr große Feldlängen zuläßt. Trotzdem entspricht sie nicht den eben beschriebenen Spezifikationen an "strukturorientierte Volltextsysteme“.

\section{Lernprogramme}

Das Regelwissen, von dem eben im Zusammenhang mit den juristischen Expertensystemen die Rede war, ist zugleich Gegenstand des juristischen Lernens. Von daher ist es leicht verständlich, daß die auf die Emwicklung von Expertensystemen bezogenen Arbeitsprojekte ebenfalls im Bereich der Lernprogramme ak-

(15) Die Ermäßigung beruht z.T. (wie etwa im Falle Nordrhein-Westfalens) auf Rahmenabkommen mit juris. Darüber hinaus bietet juris Universitäten einen Ausbildungsrabatt an, wenn sie Veranstaltungen anbieten, die den Umgang mit juris zum Inhalt haben.

(16) Vgl. zu den Zahlen für nordrhein-westfälische Universitätsbibliotheken Rütger Schäfer, Online-Informationsvermittlung 1986 in Nordrhein-Westfalen, Mitteilungsblatt des Verbandes der Bibliotheken des Landes Nordrhein-Westfalen, 2/1987, S. 194-197.

(17) Genannt sei etwa der Test von ZYINDEX am CIPPool der juristischen Fakultät Münster.

(18) Ein wichtiger Schritt in diese Richtung ist darin $\mathrm{zu}$ sehen, daß das u.a. bei juris verwendete GOLEM-System jetzt auch auf einem $P C$ verfügbar ist. 
tiv waren. (19) Auf dem Gebiet der juristischen Lernprogramme markieren die Arbeiten der amerikanischen CAL(Computer Assisted Learning)-Vereinigung und ihrer englischen Mitgliedsgesellschaft den Stand der Technik. Zu erwarten ist, daß für die Datenstruktur und den Aufbau von Lernprogrammen durch die Arbeiten dieser Vereinigung ein Standard gesetzt werden wird, was für die Vereinheitlichung der Entwicklungsumgebung und den Datenaustausch sehr wichtig ist.

Die bisherigen Entwürfe für Lernprogramme zum deutschen Recht sind noch nicht sehr zahlreich. (20) Immerhin besteht aber ein Kontakt zur englischen CAL-Vereinigung, der hoffen läßt, daß die entsprechenden Arbeiten sich ausweiten werden.

Es wäre sicher voreilig, Prototypen von Lernprogrammen am Maß des theoretisch Möglichen und Wünschbaren zu messen. Wie auch bei den anderen besprochenen Technologien besteht jedoch im Feld der Lernprogramme die Gefahr, daß das neue Medium nicht seinen Möglichkeiten entsprechend adäquat eingesetzt wird. Lernprogramme etwa, die im wesentlichen dazu führen, Texte auf dem Bildschirm zum Lesen zu präsentieren, sind dem Buch nicht nur in keiner Weise überlegen, sondern im Grunde genommen eine Regression hinter den erreichten Stand der Buchtechnologie. Dem Buch überlegen sind aber diejenigen Lernprogramme, die den Lernenden. „räumlich“ durch Wissensstrukturen führen, die in der Abbildung des Wissens in die Ebene der Buchseite nicht zugänglich sind, und dies selbst dann nicht, wenn man mit gedruckten Diagrammen arbeitet. Außerdem sollte bei Lernprogrammen eine professionelle Fundierung in dem Sinne hinzukommen, daß die Lernumgebung der späteren Arbeitsumgebung entspricht. (21)

\section{Desktop Publishing}

An der Schnittstelle von Textverarbeitung und Buchproduktion ist das „Desktop Publishing“ angesiedelt. Darunter versteht man gegenwärtig, daß mit Hilfe von Laserdruckern mikrocomputergestützt Druckwerke erzeugt werden, die im Layout gesteigerten Ansprüchen genügen. Im juristischen Bereich sind neben normalen Manuskripten zuerst Gutachten in dieser qualitativ besseren Form publiziert worden. Wegen der kostengünstigen Möglichkeiten der Herstellung zeichnet sich ab, daß wissenschaftliche Literatur in Kleinstauflagen auf diese Weise in ansprechend lesbarer Form am besten herzustellen ist. Es wird also über kurz oder lang „Newsletter ${ }^{“}$ der verschiedensten Art geben, die „desktop“ produziert neben Zeitschriften der traditionellen Art treten.

\section{Computergestützte Buchproduktion}

Der Weg aus der Textverarbeitung in den Fotosatz kann mittlerweile ebenfalls als etabliert betrachtet werden. Zwar kommt es verschiedentlich noch wegen unzureichender Projektplanung zu Reibungsverlusten, die die bei diesem Verfahren möglichen Vorteile für den Autor manchmal nahezu gänzlich aufzebren. Das ist indessen als Übergangserscheinung zu betrachten, die bald der Vergangenheit angehören wird. Schon jetzt kann man beobachten, daß der im Textverarbeitungssystem geschriebene Text mit den darin enthaltenen Markierungen bei Vorliegen eines auf dieses Textverarbeitungsprogramm bezogenen Umsetzungsprogramms automatisch in den Satz übernormmen werden kann.

Wie schon bei der Textverarbeitung zeigt sich allerdings auch hier die Tendenz, den Fortschritt gewissermaßen auf dem niedrigsten möglichen Niveau festzuschreiben. Sicher ist die Überführung des einmal elektronisch vorhandenen Textes in den Satz erfreulich, da dadurch das Korrekturlesen weitgehend entfallen kann. Dadurch wird aber eine Qualitätsverbesserung des gedruckten Werks nicht erreicht. Eine Möglichkeit, sich in dieser Richtung zu bewegen, bestünde darin, die gerade bei juristischen Werken besonders wichtigen Indexe neu zu strukturieren. Es gibt hierzu bereits geeignete Unterstützungsprogramme, diese werden jedoch im juristischen Bereich soweit ersichtlich noch nicht systematisch und auf breiter Ebene ausgeschöpft.

\section{Elektronische Kommunikation}

Die Stellungnahme zu diesem aus Gründen der systematischen Vollständigkeit aufgenommenen Punkt kann kurz ausfallen: Im juristischen Wissenschaftsbereich spielen in der Bundesrepublik "Mailboxen" keine Rolle. Auch das „European Academic Research Network" (EARN) wird, soweit ersichtlich, von deutschen Juristen nicht in nennenswertem Umfang genutzt. Gleiches gilt (noch) von BTX, obwohl auf diesem bei weitem kostengünstigsten Netz bereits juristische Informationsangebote von Belang zur Verfügung stehen (22) und über den Mitteilungsdienst bzw. in Verbindung mit externen Rechnern flexibler Informationsaustausch möglich ist.

Für die Zukunft liegt im Telekommunikationsbereich der Schlüssel für eine Integration unterschiedlicher Informatik-Hilfsmittel. Zur Veranschaulichung sei nur eine mögliche Form des Zusammenwirkens genannt: Das mit der Textverarbeitung erstellte Manuskript wird in eine Datenbank übertragen. Dort steht es für Recherchen zur Verfügung, kann aber auch (etwa bei Vorliegen einer ausreichenden Anzahl von Subskriptionen) aus der Datenbank publiziert werden. Andere Forscher können jederzeit Anmerkungen zu dem Artikel in der Datenbank anbringen. Auf diese Weise kann sich elektronische Literatur und Kommunikation in umfassender Form als Ergänzung zu den bisherigen Vermittlungsmedien etablieren.

(wird fortgesetzt)

(19) $\mathrm{Zu}$ nennen ist wiederum Tübingen mit DIALTUE.

(20) Neben Tübingen (Haft) sind - soweit ersichtlich noch zu nennen München (Philipps) und Freiburg (Bund).

(21) So der wichtige Vorschlag von Jones (Leicester) beim IBM-Kongreß „EDV und Geisteswissenschaften“ (Baden-Baden 1986).

(22) Vgl. Projektgruppe „Bürokommunikation für Rechtsanwälte «/Fernmeldeamt Hagen (Hrsg.), Broschüre zum 44. Deutschen Anwaltstag. 